\title{
A Comparison of ArcGIS and QGIS for Animation
}

I compare two GIS programs which can be used to create cartographic animations - the commercial Esri Arc GIS and the free and open-source QGIS. ArcGIS implements animation through the "Time Slider" while QGIS uses a plugin called "TimeManager." There are some key similarities and differences as well as functions unique to each plugin. This analysis examines each program's capabilities in mapping time series data. Criteria for evaluation include the number of steps, the number of output formats, input of data, processing, output of a finished animation, and cost. The comparison indicates that ArcGIS has more control in input, processing, and output of animations than QGIS, but has a baseline cost of $\$ 100$ per year for a personal license. In contrast, QGIS is free, uses fewer steps, and enables more output formats. The QGIS interface can make data input, processing, and output of an animation slower.

KEYWORDS: Time Slider; TimeManager; cartographic animation; time series

\section{INTRODUCTION}

BEgINNING In THE late 1930s, the medium of film was used to create, duplicate, transport, and display animated maps (Peterson 2000). However, these methods required large amounts of time and resources to create, and distribution and display were complicated, and thus their application was still limited. Because of more recent advances in the digital storage, manipulation, and transmission of maps, animation has become more widely used in cartography.

Animated maps can either be temporal or non-temporal (Peterson 2000). Examples of temporal animation include changes in agricultural yields by year, the shifting locations of crime in a city by time of day, or the progression of weather events (Gula et al. 2015). On the other hand, a non-temporal animation might involve a fly-through (where a viewer is taken along a route by progressively stepping between frames) or a classification animation (where the same map is classified in various ways in quick succession). Some argue that all animations are temporal: even "non-temporal" animations take up a span of timethere is "presentation time" as opposed to "real time." For the purposes of this comparison, only real time temporal animations were explored.

My objective is to evaluate the differences in the creation of a time-series animation between two popular GIS programs: ArcGIS and QGIS. ArcGIS, a commercial program by Esri, is quite likely the most widely used GIS program in the United States. QGIS is open-source and supported by a large user-base of cartographers, GIS developers, and hobbyists; it is the most widely-used GIS program in the world. Both programs create animations using spatio-temporal plug-ins unique to their program architecture (Kratochvílová 2012). Previous studies have compared ArcGIS and QGIS on broad categories (Friedrich 2014), and different open-source solutions for time series mapping (Sutton and Olson 2013).

\section{THE STRUCTURE OF A TIME-SERIES DATASET}

To CREATE AN ANIMATION in either program, first a field within a dataset must be formatted as a date (Table 1). The date can have both calendar date and time information, and the format can be varied by program and for different scales of precision (from year to second). Table 1 shows an example of a time-series dataset: a Microsoft Excel 


\begin{tabular}{llllll}
\hline City & Country & Date & Tournament & Latitude & Longitude \\
\hline Yinchuan & China & $12 / 9 / 2016$ & World Cyber Arena 2016 & 38.487194 & 106.230909 \\
Boston & United States & $12 / 3 / 2016$ & The Boston Major 2016 & 42.360302 & -71.059906 \\
Los Angeles & United States & $12 / 7 / 2016$ & The Summit 6 & 34.052234 & -116.243685 \\
Montreal & Canada & $11 / 10 / 2016$ & Northern Arena BEAT Invitational & 45.500714 & -73.563254 \\
\hline
\end{tabular}

Table 1. The attribute table of a temporal dataset shapefile.

spreadsheet with latitude and longitude coordinates of tournament locations, and another field with the date each event took place. You can then export these city coordinates as a point shapefile, which can be used by both ArcGIS and QGIS. Next, the shapefile can be manipulated within each program's spatio-temporal plug-in.

\section{DATA INPUT}

\section{ARCGIS TIME SLIDER}

To activate the Time Slider plug-in, a dataset must be time-enabled. As seen in Figure 1, open the properties of the shapefile layer, navigate to the "Time" tab, and check the box next to "Enable time on this layer."

\section{QGIS TIMEMANAGER}

Enable the TimeManager plug-in through QGIS's Plugins menu (Figure 2). First-time users will have to download the plugin.

Once the TimeManager plugin is installed, it creates the TimeManager toolbar in the main project window. When the TimeManager Settings button is clicked on the toolbar (Figure 3), it brings up a new window with options for selecting the layer with time information. At the right of the window, choose "Add layer" to bring in a vector file, or "Add raster" to bring in a raster image (Figure 4). There's also the option to remove a layer that is being animated.

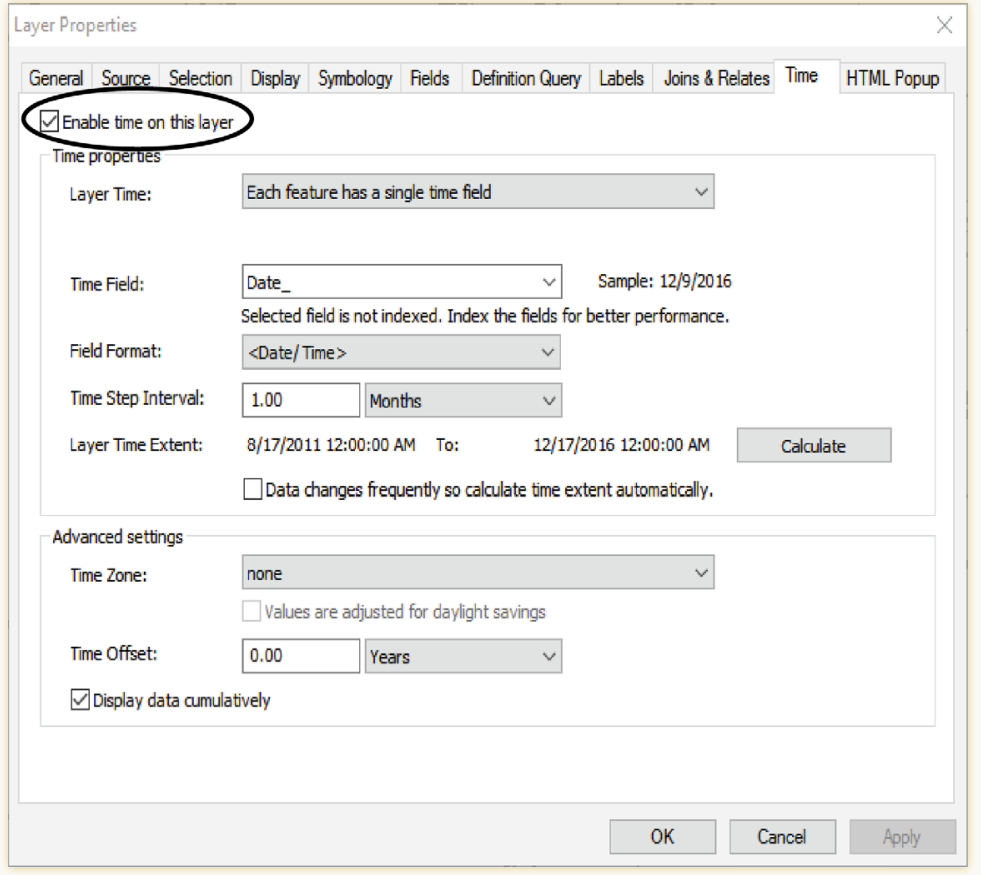

Figure 1. Enabling time on a layer in the ArcGIS Time Slider dialog box.

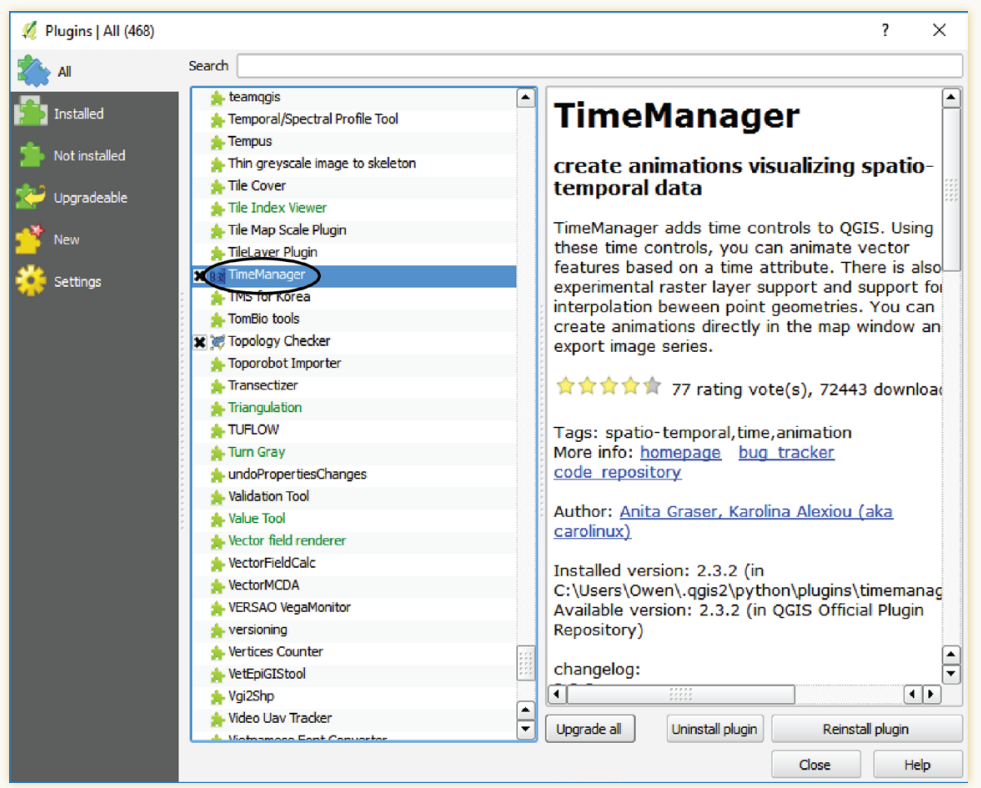

Figure 2. The QGIS Plugins menu, from which the TimeManager plugin is added. 


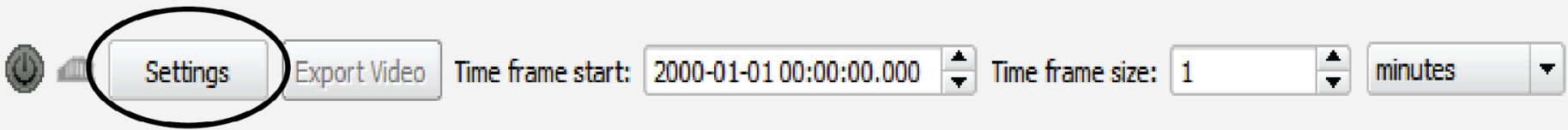

$\mathbb{W}$

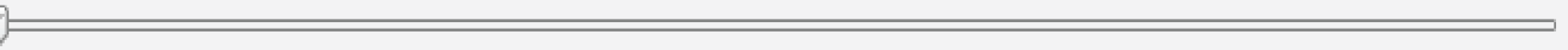

not set

Figure 3. The QGIS TimeManager toolbar. The Settings button is used to access the layer selection dialog.

\section{Time manager settings}

Layers:

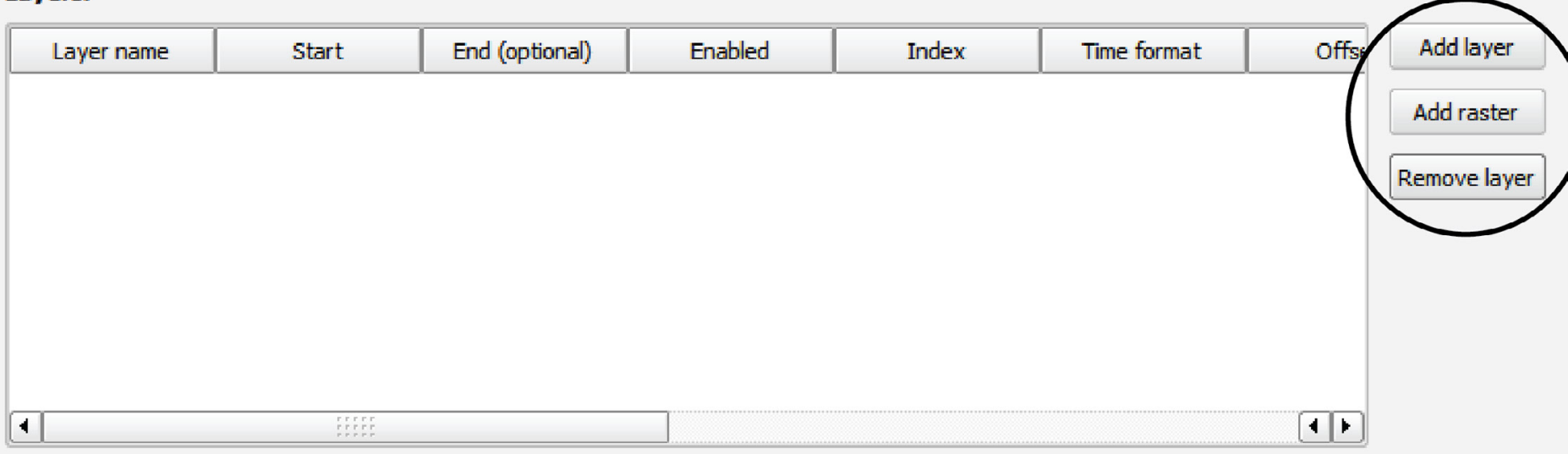

\section{Animation Options:}

Show frame for

$500 \quad \div$ milliseconds

$\square$ Play animation backwards

Looping animation

Do not export empty frames in time managed layers

$\mathbf{x}$ Display frame start time on map

Time display options

OK

Cancel

Figure 4. The Add Layer, Add Raster, and Remove Layer functions are used to choose the layer to be animated.

\section{PROCESSING OF DATA}

\section{ARCGIS TIME SLIDER}

The “Layer Time” field in Figure 1 allows either a single time for each event, or a beginning time and ending time for each event. The second option lets you show durations of events within the time-series animation. Next, select the field that has time information. Pick one field (or two, if your data have beginning/ending times) and then the format of the field. Higher degrees of precision can be specified if the data allow such accuracy. Next, decide on the "Time Step Interval." This option sets the time that passes between each frame of the animation. The 
setting will depend on the difference in spacing between the events in time: the greater the spacing, the longer the Time Step Interval needed to effectively animate the appearance.

With Time Slider, you can automatically generate the recommended Time Step Interval with the "Calculate" button. If the data needs a higher or lower number than this value, an interval can be manually entered. There is also an option to change the Time Zone, if all the events fall into a single zone. Set an offset for the time if the data were gathered in a single zone but need to be represented in another zone. The final setting in the Time tab sets the animation to display the data cumulatively, which allows the points to stay in place as new dates display new events. Otherwise, the default setting is singular, where each time interval displays its individual events, which then disappear as the new interval begins.

\section{QGIS TIMEMANAGER}

Once "Add layer" is selected, the shapefile can be chosen, along with the field in the data that indicates the Start time (Figure 5). Next, the End time is set. If the option for "No End Time" is set to "Accumulate," the animation will accumulate features as with the "display data

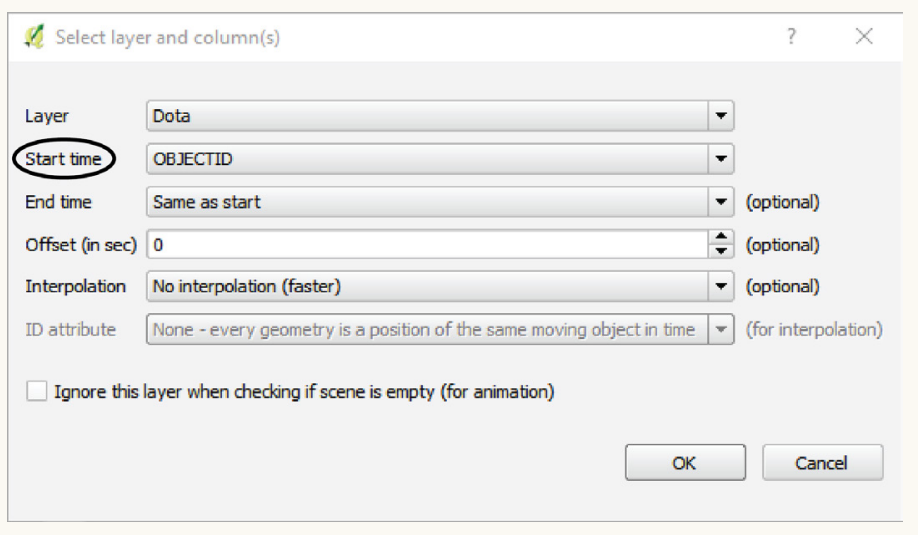

Figure 5. The QGIS Select Layers dialog, where the user must indicate the Start time.

cumulatively" option in ArcGIS. There is also an option to specify a time offset, as well as interpolation, which is a feature that ArcGIS does not implement. Interpolating values of a time series does not add any real data, as the interpolation process is not the same process that generated the other (non-missing) values in the series ( $\mathrm{Nga}$ et al. 2012), and great care is needed in employing it. If there are continuous events with long gaps between consecutive observations, these gaps can be filled with interpolated positions to get uninterrupted movement traces. Using this option will create an additional point layer in the QGIS project that contains the interpolated positions.

\section{ANIMATION SETTINGS}

\section{ARCGIS TIME SLIDER}

Once the Settings are applied, the Time Slider toolbar can be used. The Time Slider toolbar is activated by clicking the Time Slider button on the Tools toolbar (Figure 6); this toolbar is enabled by default when opening a new map document. The Time Slider toolbar is seen in Figure 7-the button on the left enables or disables time on the current map, while the one on the right button presents additional options.
The Time Slider Options dialog allows for changing the Time Display, Time Extent, Playback, and Other options. In the Time Display tab, the time zone and Time Step Interval can each be specified again or changed. The next option in the tab, Time Window (Figure 8), gives the ability to selectively display portions of the total dataset that is time-enabled. The options at the bottom of the tab to display the date and time format let you customize how

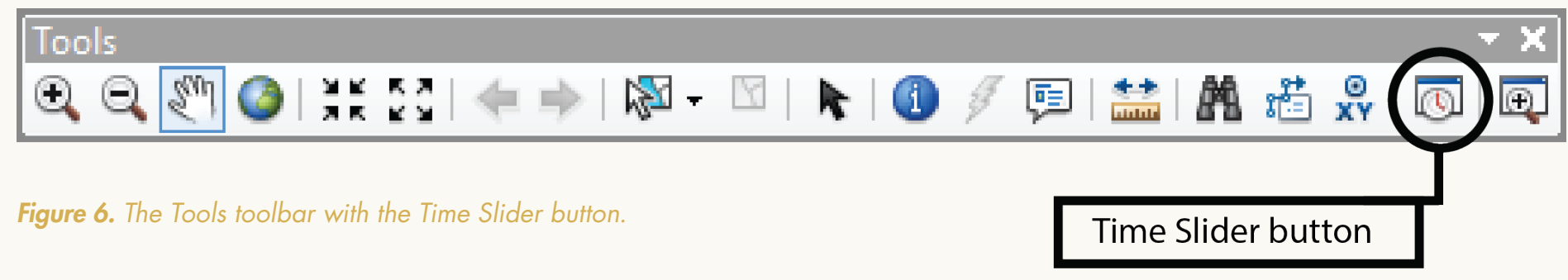




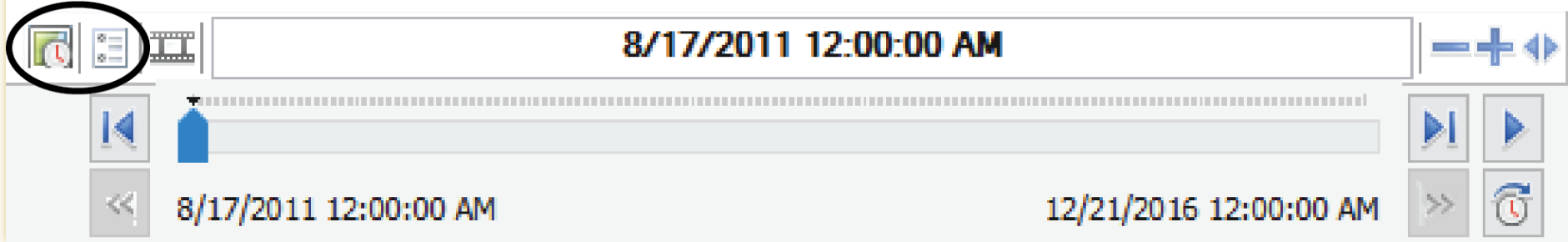

Figure 7. The Enable Time button and Time Slider Options buttons, indicated on the Time Slider toolbar.

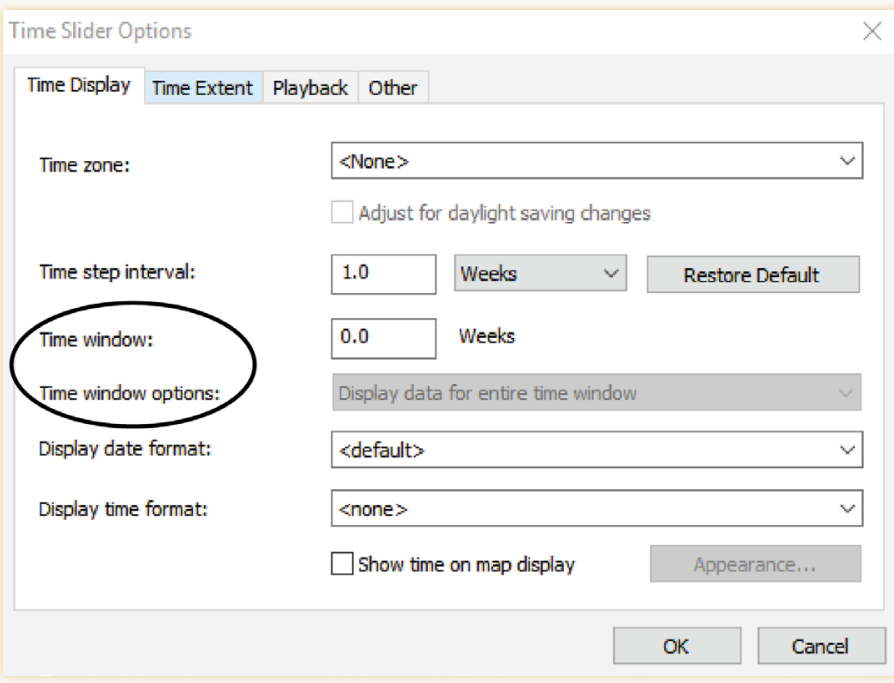

Figure 8. ArcGIS Time Slider Options dialog box with the Time Window options circled.

they appear within the Time Slider. You can also display the timestamp on the map by checking the "Show time on map display" box and customize it by clicking on the "Appearance" button.

Using the Time Extent tab (Figure 9), you can select a date range, and a start and end, from the existing data. Restrict the amount of data that is animated by using the Time Window options. This gives you the ability to display data outside the Time Step Interval, within a window that is greater than the Time Step.

The Playback tab (Figure 10) provides several options for controlling how the animation runs. You can select a slower or faster speed using the slider tool, though no specific speed can be indicated. However, you can select "Play in specified duration (seconds)" to indicate a time within which the whole animation must complete; this will adjust the frame rate. Next, the animation can be repeatedly looped, or even played in reverse, using the "After playing

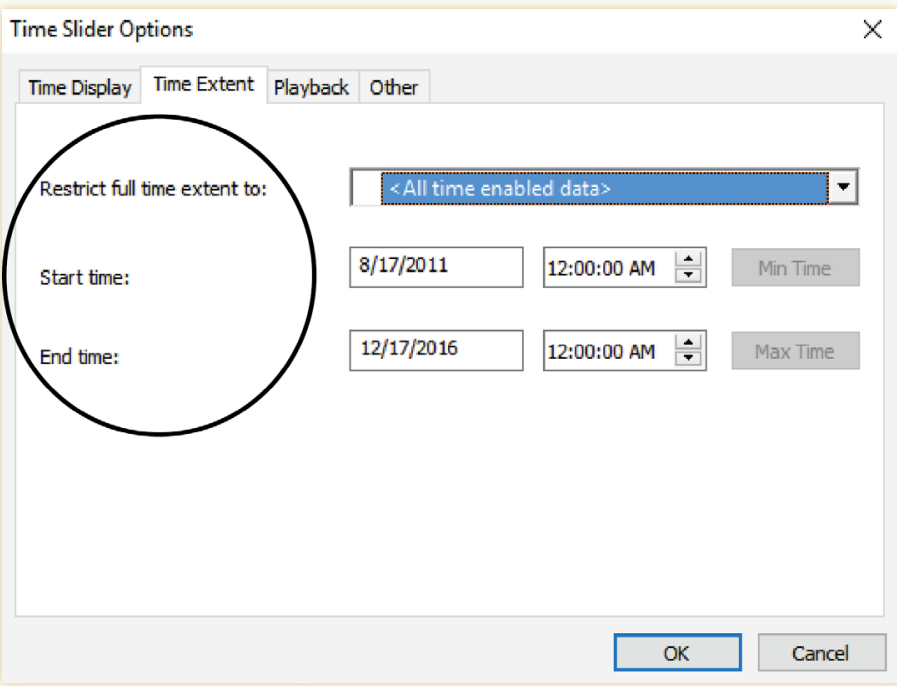

Figure 9. The Time Extent window gives the user the ability to show portions of the data.

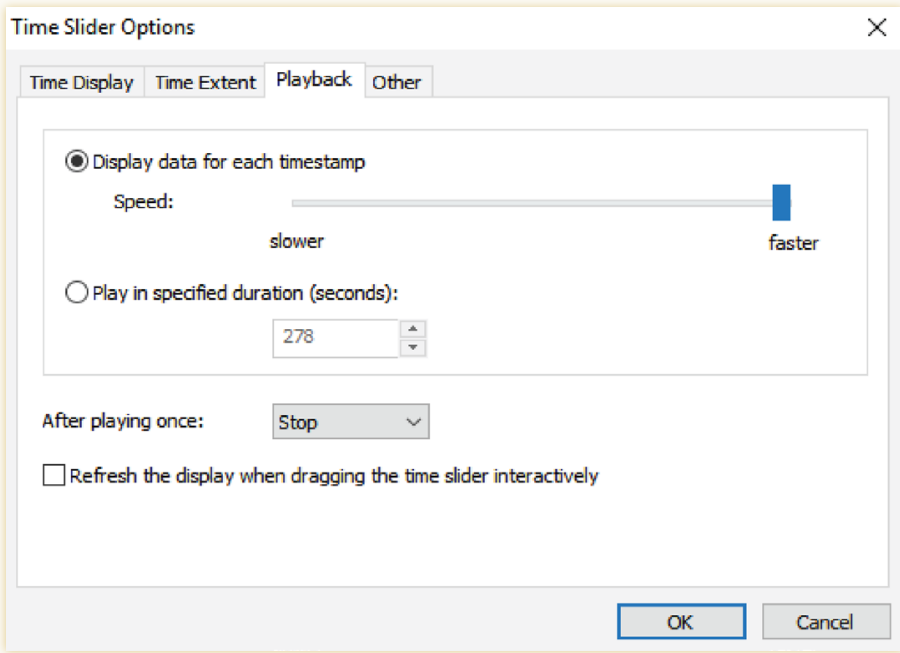

Figure 10. The ArcGIS Time Slider Playback tab allows speed, duration, repeat, and refresh settings to be changed. 


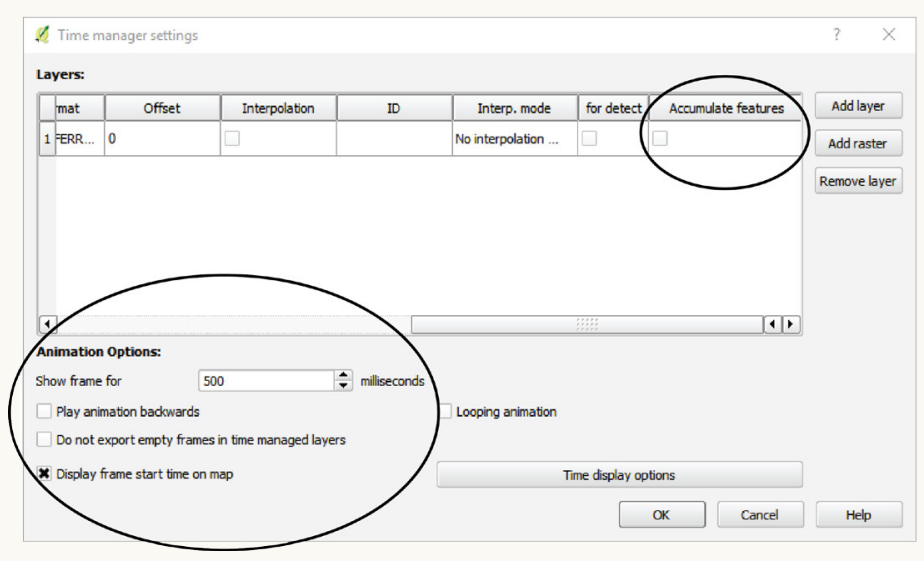

Figure 11. Animation options within the TimeManager plug-in.

once" dropdown menu. By selecting the "Refresh the display when dragging the time slider interactively" checkbox, the display will refresh automatically to show the results at the time of interaction, rather than staying static and waiting for the new input. By default, the display changes only after moving and releasing the slider.

Finally, in the Other tab, it's possible to make the Time Slider window transparent in the animation. This effect could be used if data would be obscured by the display of the Time Slider window. This feature is an example of the Time Slider's advanced animation customization.

\section{QGIS TIMEMANAGER}

QGIS likewise offers some options to customize your animation, found at the bottom of the Time Manager Settings. You can determine how long each frame will be visible, whether the animation goes in reverse, whether the animation repeats through a loop, or whether blank frames will be removed upon export of the animation (Figure 11). You can also display the frame start time on the map, which overlays text in the animation showing the date and time of each time-interval step. In the Time Display Settings window, selected by pressing the Time Display Options button, a variety of text options can be selected, including the font of the time display, the size of the font, the format of the time shown, the placement of the time display, and the time display's text color and background color. Unlike ArcMap, in QGIS, the time display cannot be made transparent in the map window.

\section{INTERACTION}

\section{ARCGIS TIME SLIDER}

The Time Slider toolbar, seen in Figure 12, gives the ability to skip to the previous time stamp, skip to the next time stamp, scroll back in time, scroll ahead in time, see the full time-extent, and increase or decrease the time extent in the animation. This allows you to customize the animation before exporting it to a video or as a series of images.

The Time Slider toolbar also provides the option to enter Live Mode, where it is possible to visualize the most recent

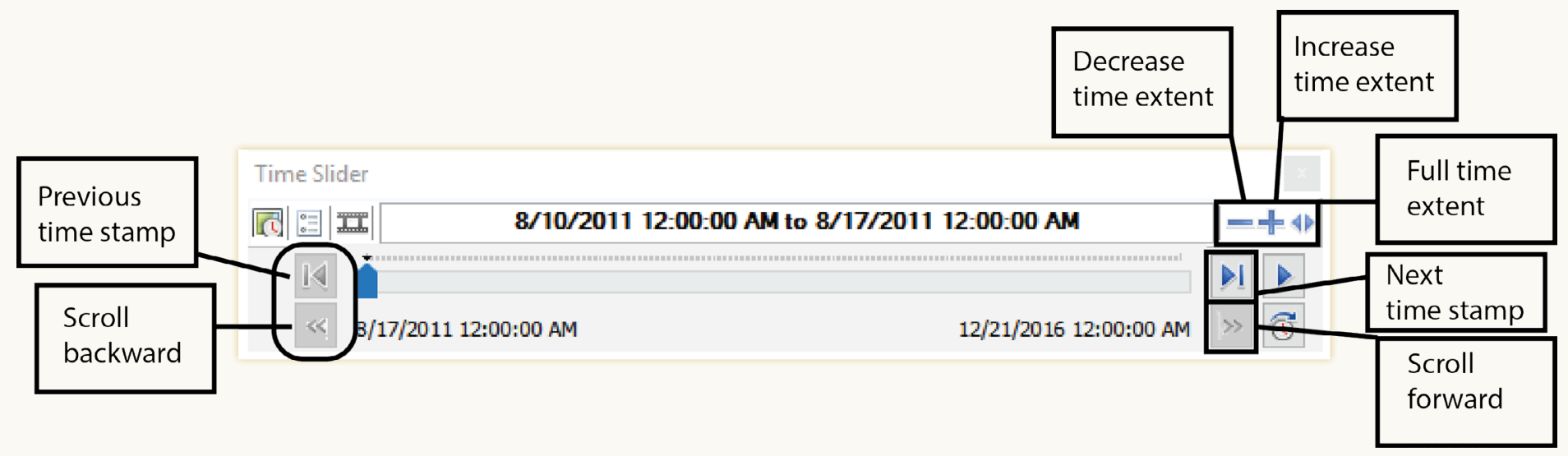

Figure 12. The Time Slider toolbar with skip next/back, scroll forward/backward, increase/decrease time extent, and full time-extent buttons. 
A1
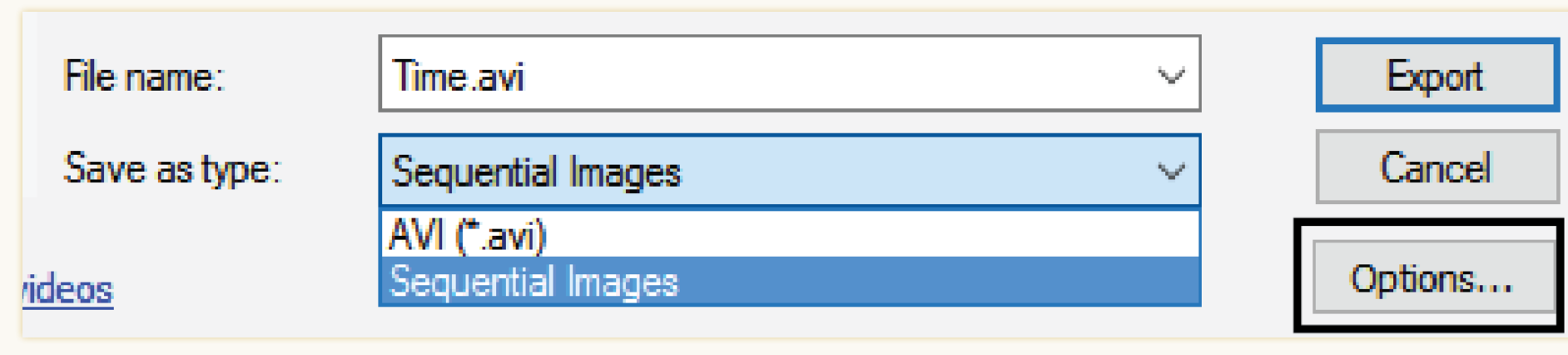

Figure 14. The ArcGIS Time Slider Export Video window, and the Options button for further customization.

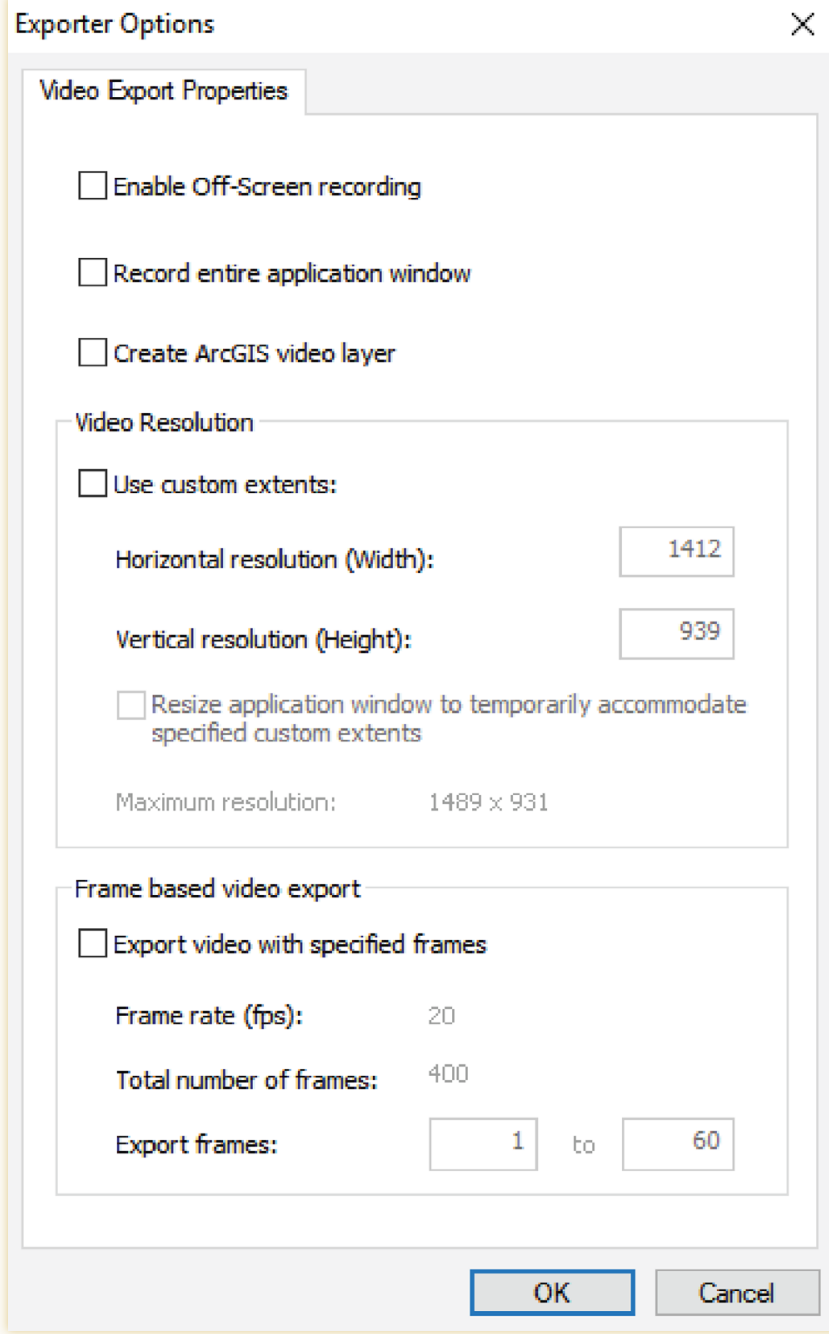

changes to the data for automatic updates. No similar feature is available in QGIS.

\section{QGIS TIMEMANAGER}

On the TimeManager toolbar (Figure 13), the extent of the data is chosen, and the size of the time frame-from microseconds to years. Also, scroll buttons and a slider enable navigation through the data.

\section{OUTPUT}

\section{ARCGIS TIME SLIDER}

The Time Slider allows the export of the animation as an .avi video file, or as sequential JPEG images, both of which can be found in a dropdown in the Export Animation dialog box (Figure 14). Sequential images can then be used in other software to create animations. The AVI video format is uncompressed, which can lead to large file sizes. In the Exporter Options menu (Figure 15), selected with the Options button, there are a variety of choices including custom video resolutions, off-screen recording, and the creation of a video layer. 
When exporting the animation as a video, select Export Video on the Time Manager toolbar, and choose either Frames only (.jpeg), Animated .gif, or Video (.mpeg) (Figure 16). This is where the animation frame delay is chosen to indicate how long the animation will stay on a frame before moving to the next frame.

A unique feature of QGIS is the Archaeological Date Time- - a button (Figure 17) on the toolbar that gives the option to display times in the XXXX BC or AD format (Figure 18), which is useful when showing an animation of data that spans the year 0 .

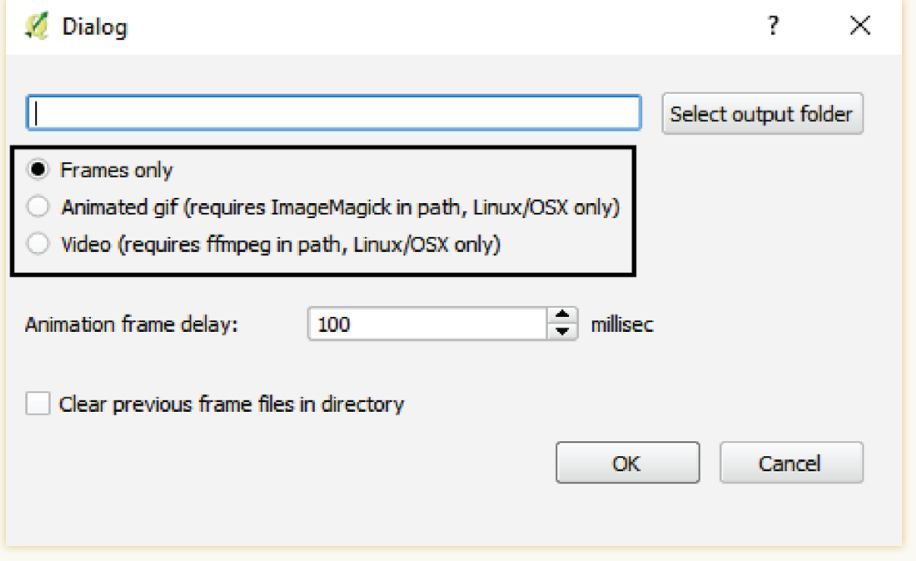

Figure 16. The QGIS TimeManager Export Video Dialog box, with the export format choices indicated.

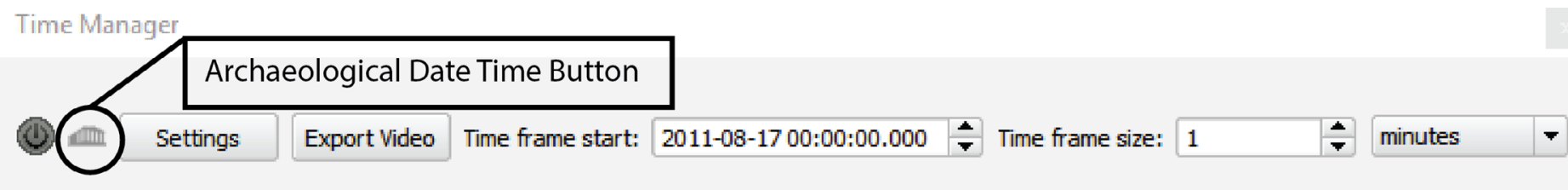

Q

Figure 17. The Archaeological Date Time Button on the Time Manager toolbar.

\section{RESULTS}

TABle 2 summarizes the comparison of ArcGIS and QGIS, while in Table 3, the unique features of each program are compared. To generate an animation, ArcGIS required more steps, offered greater control, has a higher cost, and exports in one format fewer. QGIS had fewer steps, was faster, has zero cost, and exports to more formats. In my opinion, QGIS also offered a less intuitive method for setting frame rate and timing than ArcGIS.

The ArcGIS suite is a powerful tool that is well suited for enterprise environments, and the Time Slider plugin reflects this orientation. The options for Live Mode, Interactive Updating, and Dynamic Video Layers would be beneficial for large datasets that are frequently updated, such as climate or species data. The option to record the entire window gives an analyst the ability to create a video of the entire ArcGIS program, instead of just the data view. The Enable Off-Screen recording option is

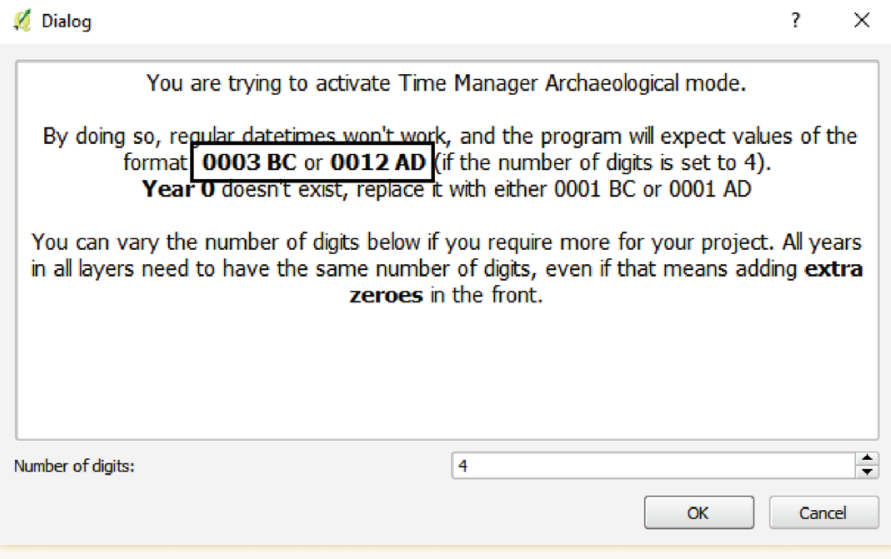

Figure 18. The QGIS Archaeological Date Time dialog box, explaining the required date format

useful to continue working while the animation is exporting. With the exception of graphs, windows opened on top of the ArcMap application will not appear in the exported video. Finally, the ability to export videos with custom 


\begin{tabular}{|c|c|c|}
\hline & ArcGIS & QGIS \\
\hline $\begin{array}{l}\text { Unique } \\
\text { Features }\end{array}$ & 8 & 6 \\
\hline Cost & $\begin{array}{l}\$ 100 \text { per year for personal } \\
\text { license }\end{array}$ & Free \\
\hline $\begin{array}{l}\text { Output } \\
\text { Formats }\end{array}$ & 2: JPEG and AVI & $\begin{array}{l}\text { 3: JPEG, GIF, } \\
\text { and MPEG }\end{array}$ \\
\hline $\begin{array}{l}\text { Video } \\
\text { Format }\end{array}$ & $\begin{array}{l}\text { AVI (uncompressed; } \\
\text { ArcGIS offers options for } \\
\text { compression) }\end{array}$ & $\begin{array}{l}\text { MPEG } \\
\text { (compressed) }\end{array}$ \\
\hline
\end{tabular}

Table 2. A summary of the time-series plug-ins' unique features, cost, output formats and video format.

resolutions, custom frame extents, and the AVI format give ArcGIS the edge over QGIS in customization.

ArcGIS uses the AVI format for video export. Video stored in AVI format is uncompressed and retains its original quality, but because there is no compression, the file size of an AVI video can be extremely large. The AVI format supports a wide range of video and audio formats and variable frame and bit rates. It does not support menus or streaming. These features make AVI an excellent format for storing and editing video, but a poor format for distributing video via the Internet or physical media. With ArcGIS, choose the compression codec when exporting AVI files to avoid the impractical file sizes generated with this format. Nearly all AVI files have an integrated codec to reduce file size.

QGIS TimeManager incorporates features that aren't offered in the ArcGIS suite, such as the option to interpolate the data during the animation, raster layer support, and the archaeological date function. QGIS's open source format and compact program size make it ideal for one-off projects and smaller GIS operations. The fact that QGIS requires fewer steps and has more output formats makes

\begin{tabular}{|c|c|c|}
\hline Features & ArcGIS & QGIS \\
\hline Step Interval & $x$ & $x$ \\
\hline Cumulative Display & $x$ & $x$ \\
\hline Time Offset & $x$ & $x$ \\
\hline Time Display & $x$ & $x$ \\
\hline Colored Display/Fonts & $x$ & $x$ \\
\hline Frame Spacing & Auto/Manual & Manual \\
\hline Font Formatting & $x$ & $x$ \\
\hline Animation Loop/Reverse & $x$ & $x$ \\
\hline Time Zone/Extent/Window & $x$ & \\
\hline Transparency of Display & $x$ & \\
\hline Interactive Display Updating & $x$ & \\
\hline Live Mode & $x$ & \\
\hline Video Layer & $x$ & \\
\hline Record App Window/Off Screen & $x$ & \\
\hline Custom Resolution/Frame Export & $x$ & \\
\hline Interpolation & & $x$ \\
\hline Raster Layer Support & & $x$ \\
\hline Archaeological Date/Time Option & & $x$ \\
\hline Empty Frame Erasure & & $x$ \\
\hline
\end{tabular}

Table 3. Summary of features between ArcGIS Time Slider and QGIS TimeManager.

it efficient for producing simple animations. QGIS also uses the more portable MPEG video format. MPEG is an older, compressed video file format that is commonly used to distribute video on the Internet and DVDs.

\section{CONCLUSION}

Animated maps ARE a powerful medium for the communication of change in mapped phenomena over time. Both Esri's ArcGIS and QGIS effectively animate time. ArcGIS has more control over the animation, but is costlier. QGIS offers a faster method with less customization and has options for interpolation and archaeological dates. For cost and simplicity, QGIS is an impressive alternative to ArcGIS for the creation of temporal animations. 
Friedrich, Corinna. 2014. "Comparison of ArcGIS and QGIS for Applications in Sustainable Spatial Planning." Master's thesis, University of Vienna.

Gula, Tănase, Cristina Grosu, Diana Nănuți, Mariana Mocanu, and Sorin N. Ciolofan. 2015. "ArcGIS Based Visualization Tool for Assessment of Earthquakes Impact." In 2015 9th International Conference on Complex, Intelligent, and Software Intensive Systems, 308-313. Blumenau, Brazil: IEEE. doi: 10.1109/ CISIS.2015.43.

Kratochvílová, Anna. 2012. "Visualization of SpatioTemporal Data in GRASS GIS.” Master's thesis, Czech Technical University in Prague.
Nga, Dao Viet, Ong Hang See, Do Nguyet Quang, Chee Yung Xuen, and Lai Lee Chee. 2012. "Visualization Techniques in Smart Grid.” Smart Grid and Renewable Energy 3 (3): 175-185. doi: 10.4236/sgre.2012.33025.

Peterson, Michael. 2000. "Cartographic Animation." Accessed June 24, 2017. http://maps.unomaha.edu/ $\mathrm{mp} /$ articles/cartographicanimation.html.

Sutton, N. Lynnae, and Jackie Olson. 2013. “Time in Open Source GIS Web-based Visualizations.” Acessed August 7, 2017. http://www.gla.ac.uk/media/ media_401759_en.pdf. 